\title{
Nucleon Transition Form Factors at JLab: Status and Outlook
}

\author{
R. W. Gothe ${ }^{1}$ \\ 1 (University of South Carolina, Columbia, SC29208, USA)
}

\begin{abstract}
The measurements of exclusive single-meson and double-pion electro-production cross sections off the proton to study nucleon resonances will be extended to higher momentum transfers with the CLAS12 detector and the energy upgraded CEBAF beam. Based on new theoretical developments to extract and interpret the electromagnetic transition form factors and on the experience gained from the most recent results, the newly formed collaboration of experimentalists and theorists shall enable us to provide unprecedented highprecision data, high-quality analyses, and state-of-the-art model and QCD based calculations in a $Q^{2}$ domain up to $10 \mathrm{GeV}^{2}$.

For the first time nucleon resonance structures will be studied at still unexplored distance scales, where the dressed quark contributions are the dominating degrees of freedom and their strong interaction is responsible for the ground and excited nucleon state formation. These studies also open up a promising opportunity to understand the origin of more than $98 \%$ of the nucleon mass that is created by strong fields predominantly at these distance scales by dressing the current quarks.
\end{abstract}

Key words baryon structure, strong degrees of freedom

PACS 13.40.Gp, 13.60.Le, 14.20.Gk

\section{Introduction}

Nucleons and, more generally, baryons have played an important role in the development of the quark model and of QCD. The concept of quarks itself was manifested by the study of baryon resonances. For many years the properties of the ground state and the excited states of baryons had been treated in terms of isobars or constituent quarks. However, currently we are at the threshold of measuring ${ }^{[1]}$, describing, and comprehending these states in terms of evolving effective degrees of freedom based on fundamental QCD ${ }^{[2-5]}$. The special importance of baryons is emphasized by recent QCD calculations on the lattice ${ }^{[6]}$ that show evidence for a "Y-shape" color flux configuration, indicating a genuine three-body force for baryons with stationary quarks and not a dominant two-body force that would generate a " $\Delta$ shape" color flux configuration. Although the flux tube picture might likely breakdown for dynamical light quarks, three-body forces remain, especially at intermediate distance scales, an essential QCD feature that can be best studied in the three-quark baryon system. Laboratories worldwide are provid- ing, and particularly their anticipated upgrades will soon provide, complementary hadronic or electromagnetic probes in the best suited energy range to perform precision experiments that test the nature of the strong force in this intermediate confinement regime. One of the leading laboratories in this research field is Jefferson Lab (JLab), where some of the most pressing experiments are planned and carried out. Examples reported here will focus on the advantages of electro-excitations in exclusive single- and doublepion production channels and on baryon transition form factor measurements to investigate the transition from meson-baryon to constituent quark degrees of freedom of the strong interaction and the undressing of those constituent quarks.

\section{Exclusive single-meson electro-pro- duction}

A large portion of the nuclear physics community enthusiastically started to investigate baryon resonances as new optimized detector systems with large solid angle and momentum coverage like CLAS and

Received 16 July 2009

1) E-mail: gothe@sc.edu

(C)2009 Chinese Physical Society and the Institute of High Energy Physics of the Chinese Academy of Sciences and the Institute of Modern Physics of the Chinese Academy of Sciences and IOP Publishing Ltd 
new high-intensity continuous electron beams like at JLab became available. The high versatility of the provided electromagnetic probes, that have negligible initial state interaction, have produced intriguing results ever since. It was realized that the isoscalar or isovector, and the electric, magnetic or longitudinal nature of the coupling to hadronic matter, probes different aspects of the strong interaction. However, the desired versatility of the electromagnetic probe comes with the complication that it mixes all these different coupling amplitudes, each with various resonance and background contributions, simultaneously into the measured cross sections.
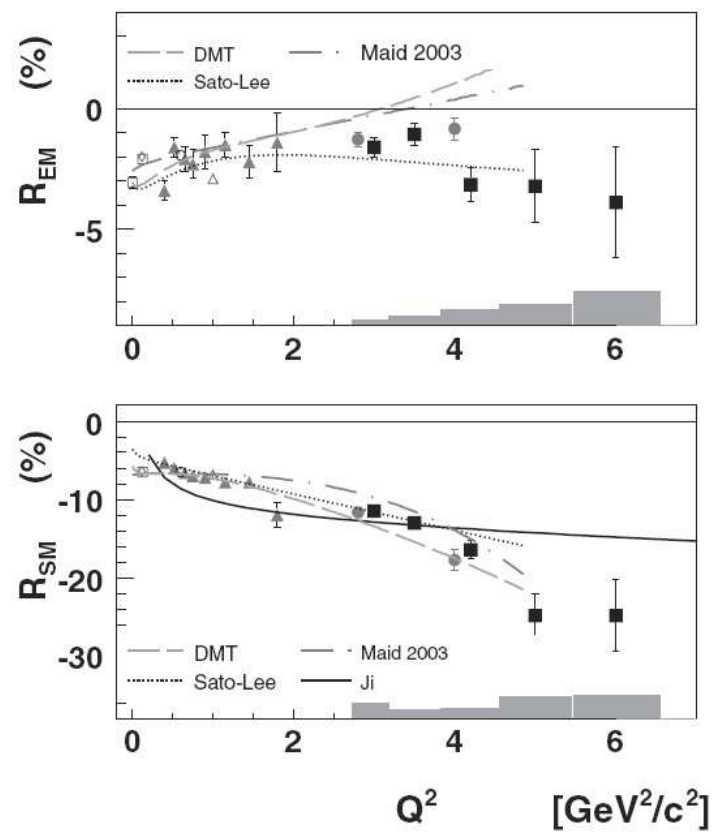

Fig. 1. The $N$ to $\Delta(1232)$ transition form factor ratios $R_{E M}$ (upper panel) and $R_{S M}$ (lower panel) ${ }^{[7]}$. The experimental results are from JLab, MAMI, ELSA, and Bates ${ }^{[8-13,16]}$.

The ability to resolve the interfering amplitudes has already been demonstrated in the case of the $N$ to $\Delta(1232) P_{33}$ transition at low intermediate momentum transfers of $0.2(\mathrm{GeV} / \mathrm{c})^{2}<$ $Q^{2}<1.0(\mathrm{GeV} / \mathrm{c})^{2}$, where the small resonant scalar quadrupole $\left(R_{S M}\right)$ amplitude could be extracted with respect to the dominant magnetic dipole amplitude with an absolute systematic uncertainty of typically $0.5 \%^{[7,9,10,14]}$ (see Figures 1 and 2).

To obtain such precision results for the extraction of isolated resonance parameters, additional isospin channels and polarization observables had been measured to disentangle the individual resonant and nonresonant coupling amplitudes ${ }^{[15-17]}$. The same precision of $0.5 \%$ was achieved for $R_{E M}=-2.5 \%^{[18]}$ in photo-production for an even more complete set of observables, see Figure 1, whereas the fundamental approach of how to perform a complete experiments in pseudo-scalar photo-production is described in ${ }^{[19]}$ and such experiments are underway at JLab.

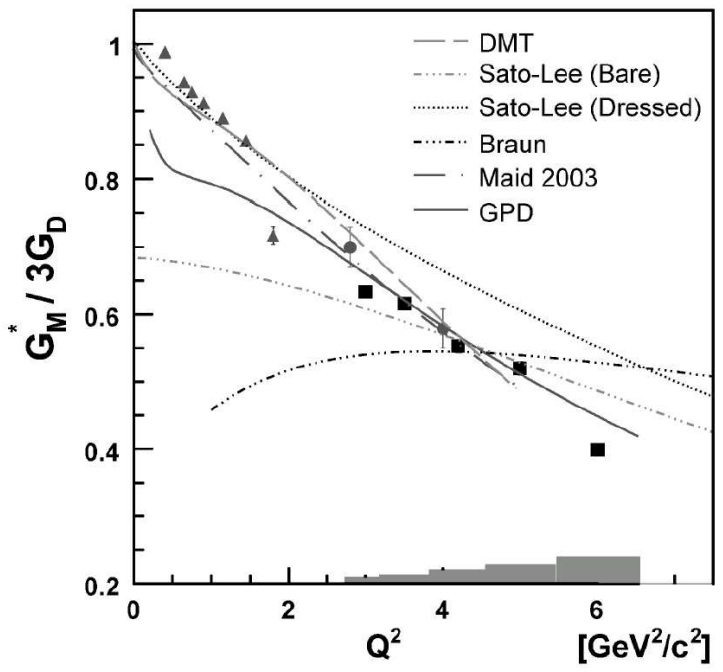

Fig. 2. The magnetic $N$ to $\Delta(1232)$ transition form factor normalized to the dipole form factor $G_{M}^{*} / 3 G_{D}{ }^{[7]}$. The experimental results are from JLab $[8,9,11]$.

In the high $Q^{2}$-limit pQCD predicts by neglecting higher twist contributions a $1 / Q^{4}$ fall-off of $G_{M}^{*}$, a $R_{E M}$ of +1 , and a $Q^{2}$ independent $R_{S M}$. The experimental results, which are now available up to $6(\mathrm{GeV} / \mathrm{c})^{2}$, as shown in Figures 1 and 2, reveal no indication of the predicted behavior in any of the three cases, but rather follow the same overall trend of the established results in the lower $Q^{2}$ region. This is particularly striking in the case of the dominating magnetic $N$ to $\Delta(1232) P_{33}$ transition form factor $G_{M}^{*}$, where already naive constituent counting rules would demand a $1 / Q^{4}$ dipole form; as well as in the case of the $R_{E M}$, that is defined by a combination of the helicity conserving amplitude $A_{\frac{1}{2}}$ and the helicity nonconserving amplitude $A_{\frac{3}{2}}$, where the simple argument of helicity conservation at high momentum transfers demands $A_{\frac{3}{2}} \ll A_{\frac{1}{2}}$, which directly leads to the prediction that $R_{E M} \rightarrow+1$. Thus the obvious questions arise: at which four momentum transfers should constituent counting rules, helicity conservation, and a pQCD based description start to dominate? Perhaps momentum transfers of up to $6(\mathrm{GeV} / \mathrm{c})^{2}$ are still not high enough.

We may attempt to deduce an answer from lattice (LQCD) ${ }^{[20]}$ or Dayson-Schwinger-Equation (DSE) ${ }^{[21,22]}$ calculations of the dynamically generated quark mass $M$ as function of the quark propa- 
gator four momentum $q$ and the basic fact that helicity should be conserved when the momentum of the hadron is large compared to its mass, $q \gg M$. In contrast to the momentum transfer, which has to be shared between all three quarks in exclusive reactions, the angular momentum transfer in resonance excitations can either involve several quarks and more complicated configurations or in principle also only one quark.

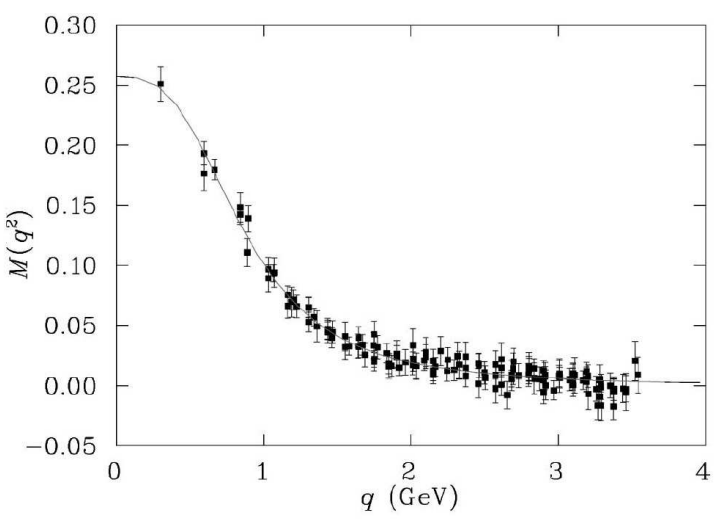

Fig. 3. Lattice QCD calculation ${ }^{[20]}$ of the quark mass in the chiral limit (data points), where $q$ is the momentum variable of the treelevel quark propagator using the Asqtad action. Similar results have been obtained in the Dyson-Schwinger approach (solid line) ${ }^{[21,22]}$ and the instanton framework ${ }^{[23]}$.

The quark mass function in Figure 3 gives at $q=2 \mathrm{GeV} / \mathrm{c}$ a quark mass of the order of $15 \mathrm{MeV} / \mathrm{c}^{2}$, which roughly corresponds to a momentum transfer of $Q^{2}=4(\mathrm{GeV} / \mathrm{c})^{2}$ for the simplest assumption that only a single quark absorbs the angular momentum introduced by the virtual photon. Under this assumption the condition for helicity conservation should definitely be fulfilled, but it would break down for $q \lesssim 1 \mathrm{GeV} / \mathrm{c}$, where quark mass steeply increases with decreasing quark momentum. These arguments lead to the prediction that for resonances that conserve angular momentum on the single quark level, the helicity conserving amplitude $A_{\frac{1}{2}}$ should start to dominate the helicity non-conserving amplitude $A_{\frac{3}{2}}$ as early as $Q^{2} \gtrsim 1(\mathrm{GeV} / \mathrm{c})^{2}$. This predicted behavior is indeed clearly visible for the preliminary $N(1520) D_{13}$ helicity amplitudes $A_{\frac{1}{2}}$ and $A_{\frac{3}{2}}{ }^{[24]}$, where the corresponding helicity asymmetry

$$
A_{\text {hel }}=\frac{A_{\frac{1}{2}}^{2}-A_{\frac{3}{2}}^{2}}{A_{\frac{1}{2}}^{2}+A_{\frac{3}{2}}^{2}}
$$

crosses zero at $Q^{2} \approx 0.7(\mathrm{GeV} / \mathrm{c})^{2}$. The $N(1685) F_{15}$ resonance shows a similar behavior with a zero crossing at $Q^{2} \approx 1.1(\mathrm{GeV} / \mathrm{c})^{2}$, whereas the $\Delta(1232) P_{33}$ helicity asymmetry stays negative with no indication of an up coming zero crossing ${ }^{[24,25]}$. Even more surprising are the very recent and preliminary results for the $N(1720) P_{13} A_{\frac{1}{2}}$ amplitude, which decreases so rapidly with $Q^{2}$ that the helicity asymmetry shows an inverted behavior with a zero crossing from positive to negative around $Q^{2} \approx 0.7(\mathrm{GeV} / \mathrm{c})^{2}{ }^{2[6]}$. This essentially different behavior of resonances underlines that it is necessary but not sufficient to measure form factors of the nucleon or of a well known resonance like the $\Delta(1232) P_{33}$ to even higher momentum transfers. The $Q^{2}$ evolution of exclusive transition form factors to as many resonances as reasonably possible is crucial to comprehend QCD at intermediate distance scales, where dressed quarks are the dominating degrees of freedom and to explore interactions of dressed quarks as they form various baryonic quantum states.

In the first case we need to push the measurements of exclusive observables and their theoretical description into the region where constituent quark degrees of freedom prevail. These experiments require the JLab and CLAS upgrades to $12 \mathrm{GeV}$. In the latter case preliminary results of the $Q^{2}$ dependencies of the helicity coupling amplitudes for the higher lying resonances $N(1440) P_{11}, N(1520) D_{13}$, and $N(1535) S_{11}$ have already been extracted up to $4.5(\mathrm{GeV} / \mathrm{c})^{2}{ }^{[24]}$. Since especially the Roper $N(1440) P_{11}$ resonance parameters have always been notoriously hard to extract* ${ }^{*}$, the variety of different theoretical approaches to describe them is extensive and includes $q^{3}, q^{3}+q \bar{q}$ cloud, and $q^{3}+g$ hybrid quark models as well as dynamical coupled channel and $N+\sigma$ molecule models.

On the one hand, Fig. 4 illustrates the high quality of the data and analysis leading to small statistical and systematic uncertainties, and on the other hand the shortcomings and strengths of various model predictions. The low $Q^{2}$ behavior is best described by certain meson cloud models ${ }^{[39]}$, while the high $Q^{2}$ behavior is described more consistently by relativistic light-front quark models ${ }^{[34,35,38]}$, indicating that the Roper resonance is likely a radial $q^{3}$ excitation and proving that it is not a quark-gluon hybrid state as predicted ${ }^{[40]}$. In an independent approach the Excited Baryon Analysis Center (EBAC) predicts, based on a full dynamical coupled-channel analysis ${ }^{[41]}$, meson-baryon dressing (meson cloud) contribu-

\footnotetext{
*With the impressive exception of recent BES (BEijing Spectrometer) results, where the Roper resonance is directly seen in the $J / \Psi$ decay into $N^{*} \bar{N}^{[27]}$.
} 
tions that seem to bridge the gap ${ }^{[42]}$ between the relativistic light-front quark models and the measured results.
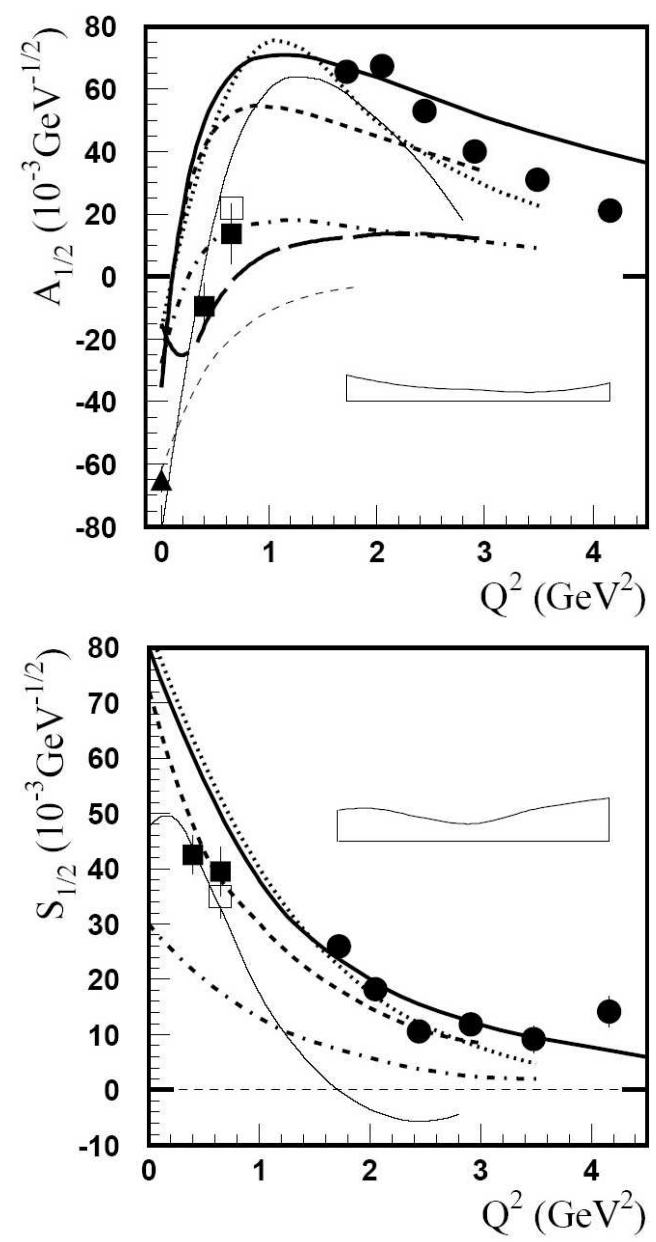

Fig. 4. Full circles are the most recent helicity amplitudes for the $N$ to Roper(1440) transition ${ }^{[28]}$ based on CLAS $\pi^{+}$electro-production data ${ }^{[29]}$. The bands represent the systematic uncertainties. The full boxes are the results obtained from CLAS data ${ }^{[30,31]}$, and the open boxes represent the results of the combined analysis of CLAS single $\pi$ and $2 \pi$ electro-production data ${ }^{[32]}$. The full triangle at $Q^{2}=0(\mathrm{GeV} / \mathrm{c})^{2}$ is the RPP estimate [33]. The bold curves correspond to relativistic light-front quark models: dotted, dashed, dash-dotted, long-dashed, and solid curves are from ${ }^{[34-38]}$, respectively. The thin solid curves are calculations obtained for a quark core dressed by a meson cloud ${ }^{[39]}$, and the thin dashed curves for a $q^{3}+g$ hybrid state ${ }^{[40]}$.

Still maybe the most interesting new result is that here, as well as in the double-pion production channel, many resonances are easier to isolate at higher $Q^{2}$ than at or close to the real photon point. The preliminary results of the extracted resonant multipole am- plitudes $M_{1-}$ and $E_{0+}$ of the Roper $N(1440) P_{11}$ and the $N(1535) S_{11}$ respectively, demonstrate how dramatically the resonance behavior of the real and the imaginary parts of these two resonant amplitudes are enhanced at higher $Q^{2}$ compared to the real photon point ${ }^{[24]}$; where the extracted resonant multipole amplitudes reflect the difficulties of isolating the Roper resonance, which even does not show any sign of a resonance peak in the differential photo-production cross sections, and of isolating the $S_{11}(1535)$, which typically had to be investigated in the $\eta$ production channel to allow a cleaner separation from neighboring resonances and background contributions.

\section{Exclusive double-pion electro-pro- duction}

The studies of double-pion production by real and virtual photons ${ }^{[43-55]}$ clearly show the capability of this exclusive channel to provide important information on $N^{*}$ electro-coupling amplitudes and hadronic decay parameters for most of the excited nucleon states. The information on $N^{*}$ parameters extracted from double-pion electro-production is complementary to that obtained in the single-pion channel. The single-pion channel is mostly sensitive to nucleon resonances in the invariant mass $W$ range below $1.7 \mathrm{GeV}^{[56]}$; while the double-pion channel also exhibits contributions from low lying $W \leq 1.6 \mathrm{GeV}$ resonance states, it becomes essential for the high lying $1.6 \mathrm{GeV} \leq W \leq 3.0 \mathrm{GeV}$ resonance states. According to the scattering data from experiments with hadronic probes ${ }^{[33]}$ as well as quark model expectations ${ }^{[57]}$, most of the high lying excited states should decay substantially or even dominantly into either $\Delta \pi$ or $N \rho$ intermediate states and thus into two pions. This makes the electromagnetic exclusive double-pion production an important tool in the investigation of nucleon resonances and the reaction dynamics as well as in the search for missing baryon states, where the term missing resonances refers to the fact that quark models based on the flavor blindness of the strong interaction predict more nucleon excitations than found experimentally.

An additional advantage of the double-pion production channel is the genuinely different nonresonant contributions, which underlines again the complementarity to the single-pion analysis. Thus, the double-pion channel is a promising way to obtain comprehensive data on the $Q^{2}$ evolution of electromagnetic form factors for most of the baryonic states. Preliminary double-pion results $[59,60]$ 
from simultaneous fits to nine single-differential cross section projections, based on a phenomenological meson-baryon model, are available for $N(1440) P_{11}$, $N(1520) D_{13}, \Delta(1620) S_{31}, N(1650) S_{11}, N(1680) F_{15}$, $N(1700) D_{13}, \Delta(1700) D_{33}, N(1720) P_{13}, \Delta(1905) F_{35}$, $N(1920) P_{33}$, and $\Delta(1950) F_{37}$ as well as an indication for a new $3 / 2+(1720)$ resonance that couples significantly weaker to $N \rho$ and stronger to $\Delta \pi$ than the already listed $N(1720) P_{13}{ }^{[33]}$.

Figure 5 shows how well the fully complementary and independent single- and double-pion results agree with each other, supporting the claim of high quality results with small overall uncertainties.
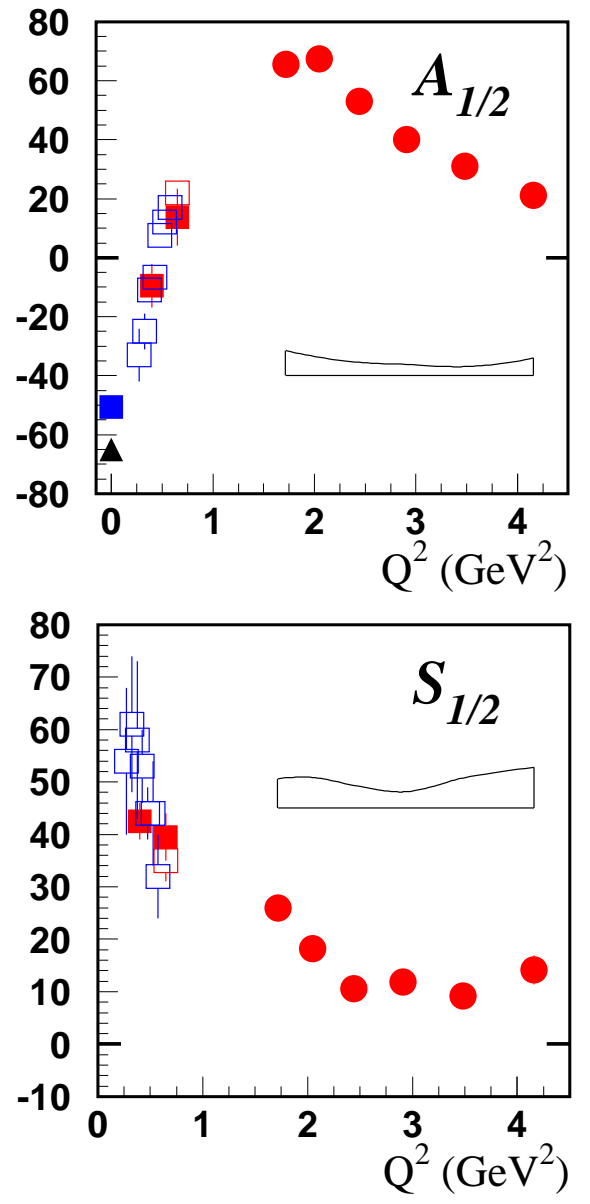

Fig. 5. Electro-coupling amplitudes off hydrogen for the $N(1440) P_{11}$ resonance in units of $10^{-3} \mathrm{GeV}^{-1 / 2}$. Red filled circles and squares are CLAS results from single-pion electroproduction data and red open squares are the combined analysis results from the singleand double-pion electro-production data, as already shown and cited in Fig. 4. Blue open squares are recent results from the double-pion production data at low $Q^{2[2,58]}$.

\section{Combined analysis}

An effective way to insure a credible separation between resonant and non-resonant mechanisms may indeed be the combined analysis of the single- and double-pion channel, which accounts for the major part of the total virtual photon cross-section in the $N^{*}$ excitation region. Since both channels have entirely different non-resonant contributions, a successful combined description of all observables measured in the single- and double-pion electro-production off nucleons with a common set of $N^{*}$ electro-coupling amplitudes and hadronic parameters would ensure a reliable separation between the resonant and nonresonant contributions in both exclusive channels. Such a successful description of all observables in both channels with a common set of $N^{*}$ electrocoupling and hadronic parameters has already been achieved in a combined analysis of the CLAS data at $Q^{2}=0.65(\mathrm{GeV} / \mathrm{c})^{2}{ }^{[32]}$ (see also Figs. 4 and 5), providing even stronger support for the phenomenological approaches ${ }^{[59,61]}$ used in the CLAS data analysis.

This work was supported in part by the National Science Foundation, the U.S. Department of Energy and other international funding agencies supporting research groups at JLab.

\section{References}

1 R. W. Gothe, V. Mokeev, et al., Nucleon Resonance Studies with CLAS12, Approved Proposal E-09003, www.physics.sc.edu/ gothe/research/pub/nstar12-1208.pdf (2009).

2 I. Aznauryan et al., Theory Support for the Excited Baryon Program at the JLab $12 \mathrm{GeV}$ Upgrade, arXiv:0907.1901v2 [nucl-th] (2009).

3 C. D. Roberts et al., these proceedings, Phys. Rev. C 67, 015203 (2003), and Prog. Part. Nucl. Phys. 33 (1994).

4 H.-W. Lin et al., these proceedings, arXiv:0810.5141 [hep- lat] (2009), and Phys. Rev. D 79, 114505 (2009).

5 V. M. Braun et al., Phys. Rev. D 74, 074501 (2006), Phys. Rev. D 73, 094019 (2006), and Phys. Rev. D 65, 074011 (2002).

6 H. Ichie, V. Bornyakov, T. Steuer, and G. Schierholz, heplat/0212024 (2002).

7 M. Ungaro et al., Phys. Rev. Lett. 97, 112003 (2006).

8 I. G. Aznauryan, Phys. Rev. C 67, 015209 (2003).

9 K. Joo et al., Phys. Rev. Lett. 88, 122001 (2002).

10 R.W. Gothe, Proceedings of NSTAR 2002, World Scientific (2003).

11 V.V. Frolov et al., Phys. Rev. Lett. 82, 45 (1999). 
12 C. Mertz et al., Phys. Rev. Lett. 86, 2963 (2001).

13 R. Beck et al., Phys. Rev. C 61, 035204 (2000).

14 G. Laveissiere et al., Phys. Rev. C 69, 045203 (2004).

15 K. Joo et al., Phys. Rev. C 70, 042201 (2004).

16 J.J. Kelly et al., Phys. Rev. Lett. 95, 102001 (2005).

17 H. Egiyan et al., Phys. Rev. C 73, 025204 (2006).

18 R.A. Arndt et al., nucl-th/9708006 (1997) and Phys. Rev. C 66, 055213 (2002).

19 I.S. Barker, A. Donnachie, and J.K. Storrow, Nucl. Phys. B 95 (1975).

20 P.O. Bowman et al., Phys. Lett. D 66, 014505 (2002) and arXiv:hep-lat/0209129 (2002).

21 M.S. Bhagwat et al. arXiv:nucl-th/0304003 (2003).

22 H. Iida et al., Nucl. Phys. Proc. Suppl. 141 (2005).

23 D. Diakonov and V. Petrov, Phys. Lett. B 147 (1984).

24 Preliminary JLab Results, K. Park and I. Aznauryan, Private Communication (2008)

25 L. Tiator et al., these proceedings, Eur. Phys. J. A 34 (2007), Nucl. Phys. A 645 (1999).

26 Preliminary JLab Results, V. Mokeev, Private Communication (2009).

27 M. Ablikim et al., Phys. Rev. Lett. 97, 062001 (2006).

28 I. Aznauryan et al., arXiv:nucl-ex/0804.0447 (2008).

29 K. Park et al., Phys. Rev. C 77, 015208 (2008).

30 I.G. Aznauryan et al., Phys. Rev. C 71, 015201 (2005).

31 K. Joo et al., Phys. Rev. Lett. 88, 122001 (2002); K. Joo et al., Phys. Rev. C 68, 032201 (2003); K. Joo et al., Phys. Rev. C 70, 042201 (2003); H. Egiyan et al., Phys. Rev. C 73, 025204 (2006)

32 I.G. Aznauryan et al., Phys. Rev. C 72, 045201 (2005).

33 S. Eidelman et al., Phys. Lett. B 592 (2004).

34 S. Capstick and B.D. Keister, Phys. Rev. D 51 (1995).

35 H.J. Weber, Phys. Rev. C 41, 2783 (1990).

36 F. Cardelli et al., Phys. Lett. B 397 (1997).
37 B. Julia-Diaz, Phys. Rev. C 69, 035212 (2004).

38 I.G. Aznauryan et al., Phys. Rev. C 76, 025212 (2007).

39 F. Cano and P. Gonzalez, Phys. Lett. B 431 (1998).

40 Z. Li and V. Burkert, Phys. Rev. D 46 (1992).

41 A. Mastsuyama et al., Physics Reports 439 (2007).

42 T.-S. H. Lee et al., Theory Support for the Excited Baryon Program at the JLab $12 \mathrm{GeV}$ Upgrade, arXiv:0907.1901v2 [nucl-th] (2009), and B. Julia-Diaz et al., these proceedings.

43 M. Ripani et al., Phys. Rev. Lett. 91, 022002 (2003).

44 M. Bellis et al., Proceedings of NSTAR2004, World Scientific (2004).

45 M. Battaglieri et al., Phys. Rev. Lett. 87, 172002 (2001).

46 S. Strauch et al., Phys. Rev. Lett. 95, 162003 (2005).

47 G. Fedotov et al., CLAS Analysis Note, June 2006.

48 U. Thoma, Int. J. Mod. Phys. A 20 (2005).

49 C. Wu et al., Eur. Phys. J A 23 (2005).

50 Y. Assafiri et al., Phys. Rev. Lett. 90, 222001 (2003).

51 J. Ahrens et al., Phys. Lett. B 624 (2005).

52 M. Kotulla et al., Phys. Lett. B 578 (2004).

53 W. Langgartner et al., Phys. Rev. Lett. 87, 052001 (2001).

54 F. Harter et al., Phys. Lett. B 401 (1997).

55 A. Braghieri et al., Phys. Lett. B 363 (1995).

56 V. Burkert and T.-S.H. Lee, Int. J. Mod. Phys. E 13 (2004) and nucl-ex/0407020.

57 S. Capstick and W. Roberts, Prog. Part. Nucl. Phys. 45 (2000).

58 V. Mokeev et al., Proceedings of NSTAR2007, Springer (2008), or arXiv:0710.5616v2 [hep-ex] (2007).

59 V. Mokeev et al., arXiv:hep-ph/0512164; Phys. of Atom. Nucl. 66 (2003); Phys. of Atom. Nucl. 64 (2001).

60 Preliminary JLab Results, V. Mokeev, Private Communication (2008).

61 I.G. Aznauryan, Phys. Rev. C 68, 065204 (2003) and Phys. Rev. C 67, 015209 (2003). 